\title{
The influence of mechanical properties on wall stress and distensibility of the dilated ascending aorta
}

\author{
Ruth J. Okamoto, DSc ${ }^{a, b}$ \\ Haodong $\mathrm{Xu}, \mathrm{MD}, \mathrm{PhD}^{\mathrm{c}}$ \\ Nicholas T. Kouchoukos, MD ${ }^{\mathrm{d}}$ \\ Marc R. Moon, MD \\ Thoralf M. Sundt III, MD
}

From the Departments of Mechanical Engineering, ${ }^{a}$ Biomedical Engineering, ${ }^{\mathrm{b}}$ and Pathology ${ }^{\mathrm{c}}$ and Division of Cardiothoracic Surgery, ${ }^{\mathrm{e}}$ Washington University, St Louis, Mo; Missouri Baptist Medical Center, ${ }^{\mathrm{d}} \mathrm{St}$ Louis, Mo; and Division of Cardiovascular Surgery, ${ }^{\mathrm{f}}$ Mayo Clinic, Rochester, Minn.

Funding provided by The Whitaker Foundation (R.J.O.). Additional funding from the National Marfan Foundation (R.J.O.).

Received for publication April 15, 2002; revisions requested June 12, 2002; revisions received March 31, 2003; accepted for publication May 1, 2003.

Address for reprints: Ruth J. Okamoto, DSc, Washington University, Campus Box 1185, One Brookings Dr, St Louis, MO 63130-4899 (E-mail: rjo@me.wustl.edu).

J Thorac Cardiovasc Surg 2003;126:842-50

Copyright $\odot 2003$ by The American Association for Thoracic Surgery

$0022-5223 / 2003 \$ 30.00+0$

doi:10.1016/S0022-5223(03)00728-1
Objectives: We sought to determine how intrinsic mechanical properties of dilated ascending aorta influence in vivo distensibility and wall stress, potential contributing factors to the risk of aortic rupture and dissection.

Methods: Fresh tissue from patients undergoing ascending aortic replacement for Marfan syndrome $(\mathrm{n}=8$, age $38 \pm 14$ years), bicuspid aortic valve with associated aneurysm $(\mathrm{n}=20$, age $55 \pm 12$ years), or idiopathic degenerative aneurysm $(\mathrm{n}=$ 7 , age $64 \pm 9$ years) was analyzed for elastic properties and circumferential residual stress. Histologic specimens were graded for elastin fragmentation, cystic changes, fibrosis, and necrosis. Distensibility and wall stresses were predicted with a cylindrical mathematical model of the aorta.

Results: Elastic properties of aortic tissue were nonlinear and extensibility decreased with age. Moderate or severe elastin fragmentation was prevalent in tissue from patients with the Marfan syndrome and more common in tissue from younger patients. Model-predicted mean circumferential stress did not vary significantly with age or patient group, but increased with aortic lumen diameter and systolic blood pressure. Model-predicted distensibility decreased with age in bicuspid aortic valve and degenerative aneurysm groups. Differences in distensibility were correlated with differences in elastic properties.

Conclusions: The age dependence of elastic properties of dilated ascending aorta is consistent with that of normal aorta observed in previous studies. Increases in mean circumferential stress with blood pressure and diameter support the clinical importance of blood pressure control and serial evaluation of aortic diameter. Declining wall strength with age may increase the risk of rupture or dissection.

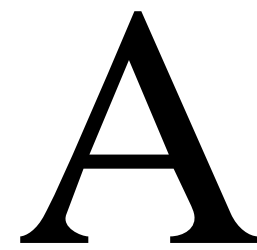

ortic rupture and dissection are mechanical failures of the aortic wall occurring with increased frequency in the dilated aorta. ${ }^{1}$ Histologic evidence of degenerative changes of the media in aneurysmal aorta have been observed in the Marfan syndrome and bicuspid aortic valve (BAV) disease., ${ }^{2,3}$ The mechanical properties of the dilated aorta in these conditions, however, have not been well characterized. We have recently reported ex vivo mechanical properties of dilated ascending aorta. ${ }^{4}$ To relate these properties to the risk of mechanical failure, we applied these findings to a cylindrical mathematical model. Using fresh tissue from patients undergoing surgical resection of ascending aortic aneurysms associated with Marfan syndrome, BAV, or degenerative disease, we performed mechanical testing to determine elastic properties ex vivo. We used these patient-specific data in a mathematical model to predict distensibility and average wall stresses under physiologic loading. Mechanical properties, predicted in vivo behavior, and histologic abnormalities were examined and compared in these 3 conditions. 


\section{Methods \\ Patients}

Ascending aorta was obtained from 35 patients (18-79 years old) undergoing graft replacement of the ascending aorta for aneurysmal dilatation at the Washington University Medical Center and the Missouri Baptist Medical Center. The study protocol was approved by the Human Studies Committees of both institutions and the patients gave informed consent. Clinical groups were defined by the presence of Marfan syndrome, BAV, or idiopathic degenerative aneurysm (DA). Patients were also grouped according to the predominant valvular hemodynamic abnormality (stenosis, regurgitation, or mixed), referred to as "valve pathology groups" hereafter.

\section{Histology}

Circumferentially oriented specimens were cut from the aortic tissue adjacent to regions used for mechanical testing, fixed in $10 \%$ formalin, embedded in paraffin blocks, and sectioned at 4- or 5- $\mu \mathrm{m}$ thickness with at least 4 serial sections obtained from each block. Histologic abnormalities were graded by the scheme of 0 (normal) to 3 (severe) proposed by Schlatmann and Becker. ${ }^{5}$ The pathologist (H.X.) was blinded to clinical data. Necrosis, defined as a focal loss of smooth muscle cell nuclei in the media, was evaluated using sections stained with hematoxylin and eosin. Cystic medial necrosis $(\mathrm{CMN})$, defined as cystic regions in the media containing mucopolysaccharides, ${ }^{5}$ was evaluated on sections stained with Movat pentachrome. Elastic fiber fragmentation and fibrosis were evaluated on sections stained with Hart's elastin and van Gieson stains, respectively.

\section{Mechanical Testing}

Aortic tissue was stored in refrigerated saline solution $\left(4^{\circ} \mathrm{C}\right)$ until testing. Mechanical testing methods have been previously described. ${ }^{4}$ Tissue was received as a short tube cut from above the sinuses of Valsalva. For most patients with the Marfan syndrome, the axial dimension of the tube was very short and a separate piece of tissue from the noncoronary sinus large enough for biaxial testing was obtained. Approximately square, biaxial test specimens (2.0-2.5 $\mathrm{cm}$ per side) with the edges aligned to the circumferential and axial directions of the aorta were subjected to equibiaxial and strip biaxial stretching protocols. Force and deformation data were combined with specimen dimensions to obtain Cauchy stresses (force/unit deformed area), stretch ratios (deformed length/unloaded length), and strains in each direction. The elastic properties were determined by fitting the experimental stress-strain data to a material law that allowed for nonlinear behavior and for anisotropy (ie, different properties in the circumferential and axial directions). Details of this law are given in the appendix. A LevenbergMarquardt optimization algorithm was used to find the best-fit material parameters for each specimen tested. When sufficient tissue was available, circumferential residual stress was characterized by measuring the opening angle ${ }^{6}$ of a 3 - to 5 -mm-wide ring. The closed ring was placed in room temperature saline solution and a radial cut was made in the ring. The opening angle, $\alpha$ (Figure $1, A)$ was defined as the angle created between the 2 end points and the midpoint of the circumference. When the intima was on the outer side of the curved ring, the opening angle was greater than $180^{\circ}$.

\section{Cylindrical Model}

We used a thick-walled cylindrical model of the aorta ${ }^{7-10}$ to predict in vivo distensibility and wall stress distribution. Although this model simplifies the aortic geometry, it incorporates important features of large strain, residual stress, and nonlinear, anisotropic elastic properties.

The stress-free configuration of the model (Figure 1, A) is an open cylinder described by inner radius $R_{i}^{*}$, outer radius $R_{o}{ }^{*}$, opening angle $\alpha$, and length $Z^{*}$. The open cylinder is closed (Figure $1, B$ ) to produce a cylinder of the same mean circumference with inner radius $R_{i}$ and outer radius $R_{o}$ and length $Z$. This compresses the inner part of the wall and stretches the outer part of the wall, producing circumferential residual stress in the unloaded cylinder. In the loaded state, the model is subject to an internal pressure $P_{i}$, and an axial stretch ratio, $\lambda_{z}$, and stretches circumferentially to a deformed outer radius $r_{o}$ (Figure 1,C). Given the unloaded radii $R_{i}$ and $R_{o}$ and the opening angle, the stress-free radii $R_{i}$ and $R_{o}$ are found by requiring the same volume and mean circumference in both states. ${ }^{9}$ For any $r_{o}$, the deformed inner radius, $r_{i}$, can also be found assuming incompressibility. The equation of radial equilibrium in cylindrical coordinates (see appendix) was integrated numerically with respect to radius to yield the internal pressure, $P_{i}$, using Matlab (Release 12, Mathworks, Natick, Mass) with 100 integration steps from $r_{o}$ to $r_{i}$.

The measured opening angle of the tissue was used in the model when available. If it could not be measured, the patient's age was used to estimate opening angle using a linear fit to experimental data previously obtained ${ }^{4}: \alpha=91.98+2.68 \cdot$ age (years), $R^{2}=0.33$. To obtain suitable $R_{i}$ and $R_{o}$ for each patient, we digitized the inner circumference of the closed rings used for the opening angle measurements and computed a mean $R_{i}$.

For some patients, particularly those with Marfan syndrome, it was known that the closed ring used for the opening angle measurement was distal to the location of the maximum aortic diameter. In these patients, we multiplied the closed ring radius by a scaling factor to obtain $R_{i}$. Preoperative transverse magnetic resonance (MR) images of the ascending aorta were digitized to obtain aortic diameters at positions in the aortic root and ascending aorta. The scaling factor was the ratio of the maximum aortic diameter to the aortic diameter at the axial location where the closed ring was cut. When MR images were not available, the scaling factor was the ratio of the diameter of the larger to smaller end of the harvested aortic tissue. For all patients, $R_{o}$ was obtained by adding the measured unloaded thickness of the biaxial specimen to $R_{i}$.

Like other blood vessels, the aorta retracts when it is removed from the patient and $\lambda_{z}$ corresponds to the amount that the vessel must be stretched to return it to the in vivo length. As we did not measure $\lambda_{z}$, we assumed $\lambda_{z}=1.2$ for all patients, the approximate mean value reported by Learoyd and Taylor ${ }^{11}$ for nondilated human thoracic aorta.

Using this input data for each patient, the model was solved at 2 internal pressures equal to the patient's reported preoperative systolic $\left(P_{\text {sys }}\right)$ and diastolic $\left(P_{\text {dias }}\right)$ blood pressures as measured by brachial cuff. The model results were used to predict the distensibility, $D$, as defined by Hirata and colleagues, ${ }^{12}$ that would be observed for patients in vivo: 


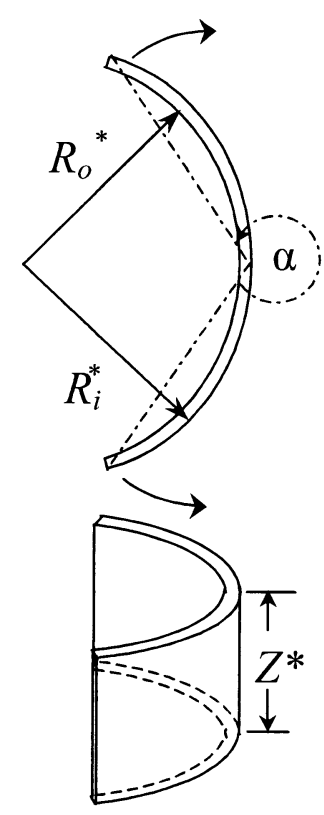

(A)
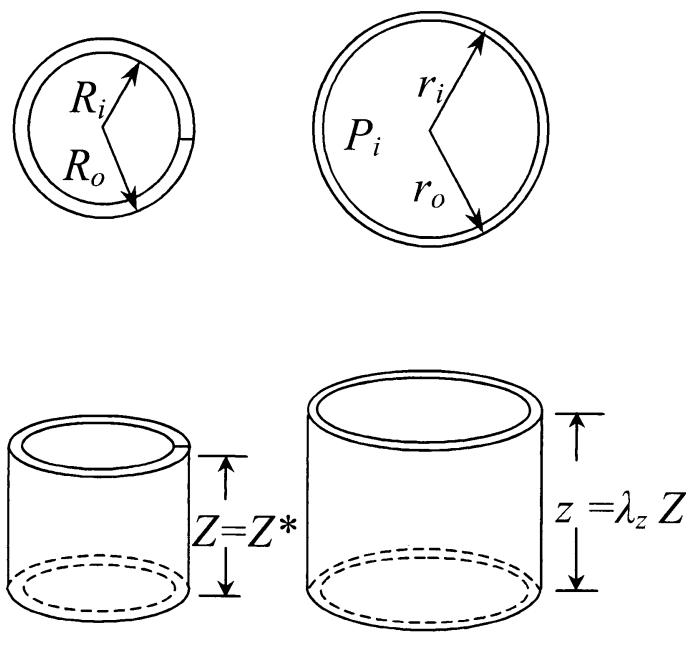

(B)

(C)

Figure 1. Cylindrical model of aorta. A, Open cylindrical segment in stress-free configuration, characterized by opening angle $\alpha$, stress-free inner radius $R_{i}^{*}$, outer radius $R_{o}^{*}$, and stress-free length $Z^{*}$. When $\alpha>180^{\circ}$ as shown, the inner radius of the closed ring becomes the outer curvature of the open ring, hence $R_{i}^{*}>R_{o}{ }^{*}$. Curved arrows show how the open segment is closed. B, Closed cylinder in unloaded configuration with inner and outer radii $\boldsymbol{R}_{i}$ and $\boldsymbol{R}_{o}$ and length $Z$. C, Deformed cylinder with applied internal pressure $\boldsymbol{P}_{i}$ and axial stretch $\lambda_{z}$ with inner and outer radii under load of $r_{i}$ and $r_{o}$.

$$
D=\frac{2\left(d_{\text {sys }}-d_{\text {dias }}\right)}{d_{\text {dias }}\left(P_{\text {sys }}-P_{\text {dias }}\right)}
$$

where the diameters $d_{s y s}$ and $d_{\text {dias }}$ are twice the model-predicted internal loaded radius, $r_{i}$, at $P_{s y s}$ and $P_{\text {dias }}$, respectively. The predicted wall thickness, $\mathrm{t}_{\mathrm{sys}}=\mathrm{r}_{\mathrm{o}}-\mathrm{r}_{\mathrm{i}}$, was computed at $P_{\text {sys. }}$. The stress distribution through the wall was averaged to obtain the mean circumferential $\left(\bar{\sigma}_{c}\right)$ and axial $\left(\bar{\sigma}_{z}\right)$ stresses. The values of $D$, $d_{s y s}, \bar{\sigma}_{c}, \bar{\sigma}_{z}$, and $t_{\text {sys }}$ were compared for different patient groups.

The effect of blood pressure on the lumen diameter and wall stresses was investigated by repeatedly solving the model for each patient at pressures, $P_{i}$, from 20 to $180 \mathrm{~mm}$ in $10 \mathrm{~mm} \mathrm{Hg}$ increments.

To compare the elastic properties between patients without the confounding influence of differences in blood pressure, wall thickness, or aortic diameter, we substituted the fitted material parameters for each patient into Equation (A.2) (see appendix) and solved for the circumferential and axial stretch ratios that corresponded to 2 stress states. These stress states corresponded to the mean values of diastolic stresses $\left(\bar{\sigma}_{c}=130 \mathrm{kPa}, \bar{\sigma}_{z}=76 \mathrm{kPa}\right)$ and systolic stresses $\left(\bar{\sigma}_{c}=262 \mathrm{kPa}, \bar{\sigma}_{z}=117 \mathrm{kPa}\right)$ found using the cylindrical model (see "Results" below). Because the simulated stress states are identical for each patient, differences in stretch ratios reflect differences in elastic properties.

Patients were included in the analysis only if they met all the following criteria: (1) we had calculated or measured values for all model inputs; (2) the equibiaxial stress-stretch curves obtained from biaxial test specimens became nonlinear; (3) the maximum stresses reached during biaxial testing were at least $30 \%$ of the predicted systolic stresses in the cylindrical model; and (4) the model-predicted systolic lumen diameter was at least $80 \%$ of the reported maximum aortic diameter. These 4 criteria were applied to minimize errors caused by extrapolating experimental data.

\section{Statistical Methods}

Measurements are reported as mean values $\pm 1 \mathrm{SD}$. Unpaired, 2-tailed Student $t$ tests were used to compare means between the 2 age groups. Single-factor analysis of variance (ANOVA) was used to compare mean values between different clinical groups and different valve pathology groups. Least-squares means differences were used for pairwise multiple comparisons after any singlefactor ANOVA that showed significance. Statistical analyses were carried out using the General Linear Model procedure in SAS 8.2 (SAS Institute Inc, Cary, NC).

\section{Results}

The clinical characteristics of the 35 patients are shown in Table 1. Patients with Marfan syndrome tended to be younger than those with degenerative disease, whereas those with BAV spanned the entire age range. Aortic diameters at surgery were similar. Patients with BAV more 
frequently had concomitant functional valve disease. Mechanical test data from 24 of the 35 patients has previously been reported. ${ }^{4}$

\section{Histology}

Histologic evidence of degenerative changes was observed in the majority of patients and was most pronounced in those with Marfan syndrome. Tissue adjacent to the region used for biaxial testing was analyzed from 32 of the 35 patients. Specimens for 1 Marfan patient (61-year-old man) and 2 BAV patients (56-year-old man, 28-year-old woman) were unavailable. The most common abnormality was elastin fragmentation, which was severe (grade 3) in 11 patients (7 Marfan, 1 BAV, 2 DA). Moderate (grade 2) or severe elastin fragmentation was more common in younger patients ( 11 of 17 , age $<55$ years, 6 of 15 , age $\geq 55$ years). Severe fibrosis was observed in only 1 specimen (Marfan) whereas moderate fibrosis was evident in 7 (4 Marfan, 1 BAV, 2 DA). Severe CMN was present in $1 \mathrm{BAV}$ and 4 Marfan patients. Severe smooth muscle cell necrosis was present in only 1 DA specimen.

\section{Wall Thickness}

Mean thickness of unloaded specimens was measured between patient groups as shown in Table 2. The difference in the mean unloaded wall thickness between age groups did not reach significance $(P=.174)$ and differences between clinical groups and valve pathology groups were not significant $(P>.2)$. Within the range of reported preoperative aortic diameters of the study group $(4.0-7.0 \mathrm{~cm})$, we found a slight decrease in unloaded wall thickness with preoperative aortic diameter that was not significant $(P>$ $.2)$.

\section{Mechanical Properties}

Elastic properties of aortic specimens for use in the cylindrical model were determined by biaxial testing. As shown in Figure 2, the elastic response to equibiaxial stretching was initially linear, followed by a rapid increase in stress as the tissue became very stiff (ie, the slope of the stressstretch curve steepened). The equibiaxial stretch ratio at which this nonlinear behavior occurred generally decreased with age in the BAV and DA groups. These findings cannot be interpreted, however, without knowledge of the range of stresses experienced under physiologic loading conditions. These were determined using the cylindrical model (see below).

Additional inputs to the cylindrical model are shown in Table 2. Because of the large age range spanned by the BAV group, data for BAV patients younger and older than the mean BAV age of 55 years are also shown separately. Patients were also grouped by functional valve pathology as shown in Table 1. Systolic blood pressure was highest
TABLE 1. Patient characteristics grouped by clinical diagnosis

\begin{tabular}{lccc}
\hline & $\begin{array}{c}\text { Marfan syndrome } \\
(\mathbf{n}=\mathbf{8})\end{array}$ & $\begin{array}{c}\text { BAV } \\
(\mathbf{n}=\mathbf{2 0})\end{array}$ & $\begin{array}{c}\text { DA } \\
(\mathbf{n}=\mathbf{7})\end{array}$ \\
\hline Age (y) & $38 \pm 14$ & $55 \pm 12$ & $64 \pm 9$ \\
Male gender & 5 & 16 & 4 \\
Maximum & $5.4 \pm 0.8$ & $5.0 \pm 0.7$ & $5.1 \pm 0.7$ \\
$\quad$ preoperative aortic & & & \\
$\quad$ diameter (cm)* & & & \\
Valve pathologyt & 5 & 3 & 3 \\
$\quad$ Neither & 0 & 5 & 0 \\
Stenotic & 3 & 7 & 3 \\
Regurgitant & 0 & 5 & 1 \\
Mixed & & &
\end{tabular}

*As reported by surgeon, or from preoperative computed tomography, magnetic resonance imaging, or echocardiography exam.

†As reported by surgeon, magnetic resonance imaging, or echocardiography exam.

among patients with DAs and tended to be higher among older (age $\geq 55$ years) patients with BAV. Opening angle was also higher in older patients as previously shown. ${ }^{4}$ The difference between opening angles measured directly from rings $\left(\alpha=242^{\circ} \pm 83^{\circ}, \mathrm{n}=19\right)$ and those obtained from the linear regression $\left(\alpha=225^{\circ} \pm 39^{\circ}, \mathrm{n}=16\right)$ was not significant $(P>.2)$. The unloaded lumen radius was determined directly from the closed ring radius for 21 patients and scaled for 14 patients (8 Marfan, 4 BAV, 2 DA).

\section{Cylindrical Model}

Mean circumferential and axial wall stresses as well as distensibility were predicted from the cylindrical model (Table 3). Given that specimens were obtained from aortas with similar diameters under physiologic conditions and similar predicted wall thicknesses, it is not surprising that differences in mean circumferential stresses between age groups did not reach significance $(P=.161)$. Differences between clinical groups were also not significant $(P>.2)$.

Mean circumferential stress was positively correlated $\left(R^{2}\right.$ $=0.469, P<.001)$ with the predicted systolic lumen diameter as shown in Figure 3, with an increase in circumferential stress of $62 \mathrm{kPa}$ for each $1-\mathrm{cm}$ increase in aortic diameter. Mean circumferential stress is plotted as a function of blood pressure (Figure 4, A) for 4 patients with predicted systolic lumen diameters of 4.0 to $4.6 \mathrm{~cm}$, thus minimizing the effect of systolic lumen diameter on the stresses. This demonstrates that the relation between blood pressure and mean circumferential stress is almost linear in the physiologic range. The mean slope of the circumferential stress-blood pressure relation for all 35 patients, computed from the difference between the cylindrical model stresses at diastolic and systolic pressures, was $2.37 \pm 0.68$ $\mathrm{kPa} / \mathrm{mm} \mathrm{Hg}$. An increase in systolic blood pressure of 26 

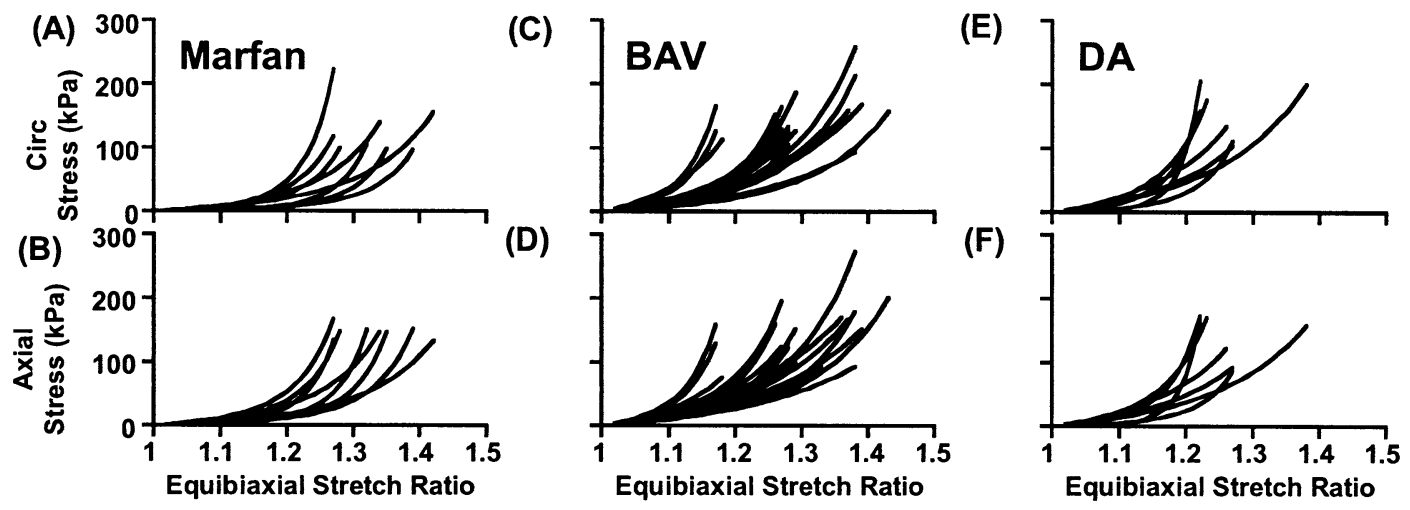

Figure 2. Comparison of response to simulated equibiaxial stretching between patient groups using fitted elastic properties obtained from biaxial test data. $A$ and $B$, Marfan. C and D, Bicuspid aortic valve (BAV). E and $F$, Degenerative aneurysm (DA). Fitted data is used to eliminate slight differences in stretching protocols between patients; however, maximum stresses shown correspond closely to stresses reached during actual equibiaxial stretching experiments.



Figure 3. Model-predicted mean circumferential stress as a function of predicted lumen diameter for all patient groups: Marfan, open square; BAV, open diamond; DA, open circle. Dashed line shows linear fit to data: $\bar{\sigma}_{c, s y s}(\mathrm{kPa})=-34.564+62.3^{*} d_{\text {sys }}(\mathrm{cm}), R^{2}$ $=0.469$.

$\mathrm{mm} \mathrm{Hg}$ thus produces a similar increase in circumferential stress as a $1-\mathrm{cm}$ increase in lumen diameter.

Because tissue from older patients was less extensible (Figure 2), axial stresses were higher among these patients at the applied axial stretch ratio $\left(\lambda_{z}=1.2\right)$. Among clinical groups, axial stress in patients with Marfan syndrome was significantly lower than for those with DAs. When pooled, patients with BAV had predicted axial stress intermediate between these groups. When stratified by age, however, younger patients with BAV had significantly lower axial stresses than older BAV patients or patients with DAs, but similar to Marfan patients.

Unlike axial stress, differences in distensibility were observed between clinical groups. Furthermore, within the


Figure 4. A, Mean circumferential stress in the cylindrical model plotted as a function of blood pressure for 4 representative patients. Marfan (39 years): open square; younger BAV (39 years): closed triangle; older BAV (66 years): open triangle; DA (77 years), closed circle. B, Normalized radius $\left(r_{f} / R_{i}\right)$ versus blood pressure for the same patients. Open symbols indicate model predicted values at $10-\mathrm{mm} \mathrm{Hg}$ pressure increments. Corresponding closed symbols indicate model-predicted values at each patient's reported preoperative systolic and diastolic blood pressure.

BAV group, younger patients demonstrated a higher distensibility than older patients. When stratified by age independent of clinical group, differences in distensibility did not 
TABLE 2. Cylindrical model input data

\begin{tabular}{|c|c|c|c|c|c|c|}
\hline & & \multicolumn{2}{|c|}{ Blood Pressure } & \multirow[b]{2}{*}{$\begin{array}{c}\text { Opening angle } \\
(\operatorname{deg} / \mu)\end{array}$} & \multirow[b]{2}{*}{$\begin{array}{c}\text { Unloaded lumen } \\
\text { diameter }(\mathrm{cm})\end{array}$} & \multirow[b]{2}{*}{$\begin{array}{l}\text { Unloaded wall thickness } \\
\qquad(\mathrm{mm})\end{array}$} \\
\hline & n & $\begin{array}{l}\text { Systolic } \\
\text { (mm Hg) }\end{array}$ & $\begin{array}{l}\text { Diastolic } \\
\text { (mm Hg) }\end{array}$ & & & \\
\hline \multicolumn{7}{|c|}{ Clinical group } \\
\hline Marfan & 8 & $115 \pm 16^{*}$ & $68 \pm 14$ & $205 \pm 51 \|, \emptyset$ & $3.4 \pm 0.6$ & $2.7 \pm 0.4$ \\
\hline BAV & 20 & $127 \pm 19$ & $73 \pm 15$ & $235 \pm 68$ & $3.2 \pm 0.5$ & $2.6 \pm 0.5$ \\
\hline$<55 y$ & 10 & $121 \pm 19 \dagger$ & $69 \pm 11$ & $185 \pm 50^{* *}$ & $2.9 \pm 0.3$ & $2.8 \pm 0.6$ \\
\hline$\geq 55 y$ & 10 & $133 \pm 17 \ddagger$ & $76 \pm 19$ & $286 \pm 39$ & $3.4 \pm 0.6$ & $2.5 \pm 0.3$ \\
\hline $\mathrm{DA}$ & 7 & $150 \pm 14$ & $80 \pm 11$ & $263 \pm 71$ & $3.3 \pm 0.6$ & $2.6 \pm 0.3$ \\
\hline \multicolumn{7}{|l|}{ Age group } \\
\hline$<55$ y & 18 & $121 \pm 18$ & $70 \pm 11$ & $193 \pm 49$ & $3.0 \pm 0.3$ & $2.7 \pm 0.5$ \\
\hline$\geq 55 \mathrm{y}$ & 17 & $138 \pm 20 \S$ & $76 \pm 17$ & $278 \pm 53+\uparrow 9$ & $3.4 \pm 0.6 \neq \ddagger$ & $2.6 \pm 0.3$ \\
\hline
\end{tabular}

Significantly different than $\mathrm{DA}:{ }^{*} P=.011, \dagger P=.016, \ddagger P=.048, \| P=.042,{ }^{*} P=.005 ;$ significantly different than $\mathrm{BAV} \geq 55$ years: $\uparrow P=.003, * * P=.0001$; significantly different than age $<55$ years: $\S P=.013, \dagger \dagger P<.0001, \ddagger \ddagger P=.014$.

TABLE 3. Cylindrical model results

\begin{tabular}{|c|c|c|c|c|c|c|}
\hline & \multirow[b]{2}{*}{$\mathbf{n}$} & \multicolumn{2}{|c|}{$\begin{array}{c}\text { Stresses at systolic blood } \\
\text { pressure }\end{array}$} & \multirow[b]{2}{*}{$\begin{array}{c}\text { Distensibility } \\
\left(\mathrm{cm}^{2} / \text { dyne }\right) \times 10^{6}\end{array}$} & \multirow{2}{*}{$\begin{array}{l}\text { Lumen diameter } \\
\text { at systolic } \\
\text { blood pressure } \\
\text { (cm) }\end{array}$} & \multirow{2}{*}{$\begin{array}{l}\text { Wall thickness at } \\
\text { systolic blood } \\
\text { pressure (mm) }\end{array}$} \\
\hline & & $\begin{array}{l}\text { Circumference } \\
\text { (KPa) }\end{array}$ & Axial (KPa) & & & \\
\hline \multicolumn{7}{|c|}{ Clinical group } \\
\hline Marfan & 8 & $257 \pm 45$ & $96 \pm 28^{*}$ & $1.43 \pm 0.68$ & $5.1 \pm 0.7$ & $1.5 \pm 0.2$ \\
\hline BAV & 20 & $257 \pm 79$ & $115 \pm 51$ & $2.21 \pm 0.83$ & $4.7 \pm 0.8$ & $1.5 \pm 0.3$ \\
\hline$<55$ y & 10 & $237 \pm 71$ & $86 \pm 17 \dagger \ddagger$ & $2.62 \pm 0.75 \|^{\prime} \#^{\prime * * *}$ & $4.6 \pm 0.7$ & $1.5 \pm 0.4$ \\
\hline$\geq 55 y$ & 10 & $277 \pm 85$ & $143 \pm 59$ & $1.79 \pm 0.72$ & $4.7 \pm 0.8$ & $1.5 \pm 0.2$ \\
\hline $\mathrm{DA}$ & 7 & $276 \pm 74$ & $135 \pm 40$ & $1.46 \pm 0.83$ & $4.6 \pm 0.8$ & $1.6 \pm 0.3$ \\
\hline \multicolumn{7}{|l|}{ Age group } \\
\hline$<55$ y & 18 & $245 \pm 60$ & $90 \pm 22$ & $2.13 \pm 0.92$ & $4.7 \pm 0.6$ & $1.5 \pm 0.4$ \\
\hline$\geq 55 \mathrm{y}$ & 17 & $278 \pm 78$ & $140 \pm 51 \S$ & $1.62 \pm 0.75$ & $4.8 \pm 0.9$ & $1.5 \pm 0.2$ \\
\hline
\end{tabular}

Significantly different than $\mathrm{BAV} \geq 55$ years: ${ }^{*} P=.019, \dagger P=.0031, \# P=.017$; significantly different than $\mathrm{DA}: \ddagger P=.0189,{ }^{* *} P=.0035 ;$ significantly different than Marfan: $\mathbb{P} P=.002$; significantly different than age $<55$ years: $\S P=.0005$.

reach significance $(P=.072)$. Distensibility decreased significantly with age in the BAV $(P<.001)$ and aneurysm groups $(P<.001)$ but not the Marfan group $(P>.2)$. Differences in distensibility $(P>.2)$ and mean stress values $(P>.2)$ between functional valve pathologies were not significant. As shown in Figure $4 B$, the relation between blood pressure and normalized lumen radius, $r_{i} / R_{i}$, is linear in the physiologic range. Although $r_{i} / R_{i}$ initially increases rapidly with blood pressure, the cylindrical model predicts that the slope of the pressure-normalized radius relation decreases before physiologic pressures are reached. The normalized lumen radius at which the slope decreases is lower in the older patients because of the difference in intrinsic elastic properties (Figure 2).

To quantify the contribution of intrinsic elastic properties to the observed differences in distensibility, we examined the calculated circumferential $\left(\lambda_{c}\right)$ and axial $\left(\lambda_{z}\right)$ stretch ratios using the fitted material properties for each patient at the simulated diastolic and systolic stress states. Both the circumferential and axial stretch ratios required to reach the simulated physiologic stresses decreased significantly with age (Figure 5, $A$ to $D$ ), and the mean values were significantly lower for patients in the older age group compared with the younger group $(P<.001)$.

Differences in mean circumferential and axial stretch ratios between clinical groups did not reach significance unless the BAV group was subdivided by age (Figure 5, E). The difference in circumferential stretch ratios between the systolic and diastolic stress states (Figure $5, F$ ) was correlated with distensibility $\left(R^{2}=0.80\right)$. This demonstrates that the model-predicted differences in distensibility between clinical groups are primarily governed by differences in the intrinsic elastic properties in the range of physiologic stresses.

\section{Discussion}

This study demonstrates that mean circumferential stress depends on the aortic diameter and systolic blood pressure but not on age or clinical diagnosis. In the absence of a compensatory increase in wall thickness with lumen diam- 
(A)

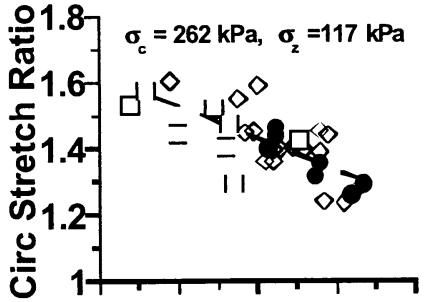

(B)

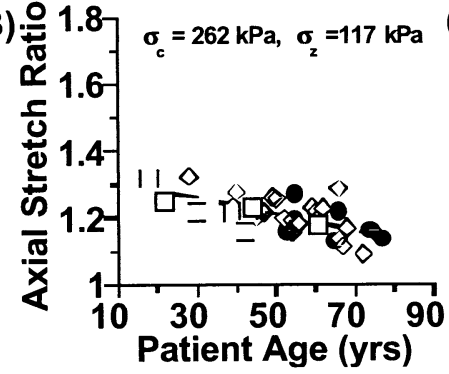

(C)

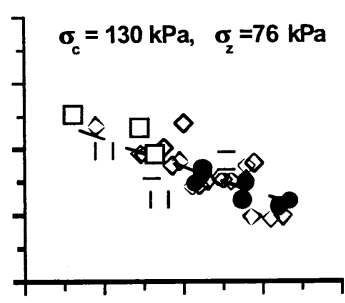

(D)

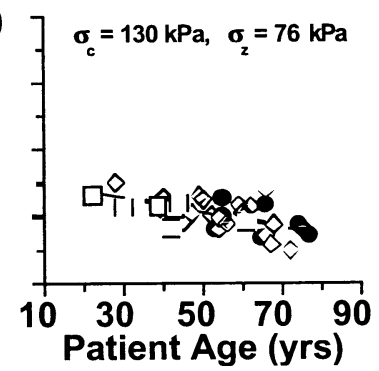

(E)

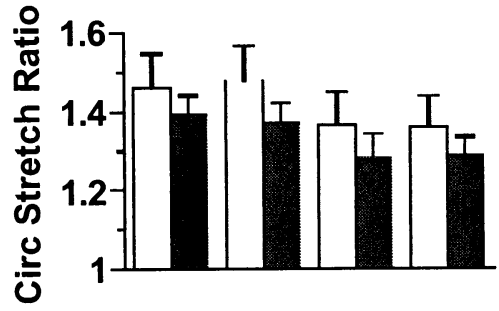

(F)

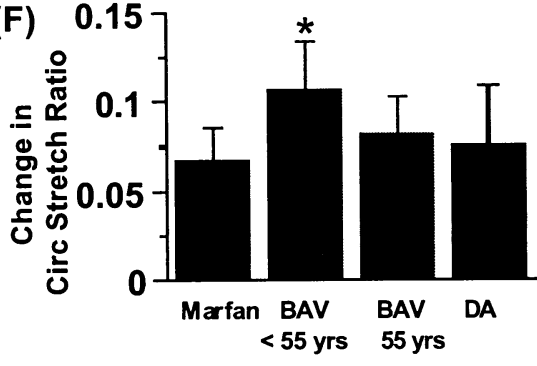

Figure 5. Stretch ratios corresponding to systolic and diastolic stress states were determined using fitted elastic properties obtained from biaxial testing. A, Predicted circumferential stretch ratios. B, Axial stretch ratios plotted as a function of age for $\sigma_{c}=262 \mathrm{kPa}$ and $\sigma_{z}=117 \mathrm{kPa}$. C, Predicted circumferential stretch ratios. D, Axial stretch ratios plotted as a function of age for $\sigma_{c}=130 \mathrm{kPa}$ and $\sigma_{z}=76 \mathrm{kPa}$. Symbols denote different clinical groups: Marfan (open square), BAV (open diamond), DA (closed circle), and dashed lines show a linear fit of the data as a function of age. E, Mean circumferential (unshaded) and axial (shaded) stretch ratios for each clinical group. $F$, Change in circumferential stretch ratio between stress states shown in $C$ and $A$. *Significantly greater change in the younger BAV group compared with all other patient groups.

eter, mean circumferential stress increased almost linearly with diameter and systolic blood pressure. Given our previous observation of reduced wall strength in older patients, ${ }^{4}$ these data suggest that mechanical failure may occur at a lower diameter in older patients. Furthermore, they support the clinical importance of blood pressure control in addition to attention to aortic diameter in reducing the risk of rupture. Indeed, the curves shown in Figures 3 and 4 demonstrate a similar incremental increase in circumferential stress with a 26-mm increase in systolic blood pressure as that for an increase of $1 \mathrm{~cm}$ in diameter. Our findings complement the clinical observations of Davies and colleagues, ${ }^{1}$ who showed that increasing thoracic aneurysm size is associated with increased yearly rate of rupture, as well as observations of Juvonen and associates, ${ }^{13}$ who identified maximum aortic diameter and advanced age as risk factors for rupture of descending thoracic aortic aneurysms.

Our data also demonstrate that among clinical groups, differences in distensibility are due primarily to intrinsic elastic properties of the aorta itself, rather than differences in aortic diameter. The mean model-predicted distensibility, $D$, of our patient groups fell slightly below the range of $D$ $=2.6$ to $2.9 \times 10^{-6} \mathrm{~cm}^{2}$ dynes ${ }^{-1}$ measured in vivo by previous investigators for young Marfan and BAV patients. ${ }^{12,14-16}$ Although distensibility was significantly lower in patients than in age-matched controls $(D=4.8$ to $6.2 \times$
$10^{-6} \mathrm{~cm}^{2}$ dynes $\left.{ }^{-1}\right)$ in these studies, aortic diameters were larger. To examine whether the larger aortic diameter could explain the differences in distensibility compared with controls, the cylindrical model was re-solved for each Marfan patient using a smaller unloaded aortic diameter while holding other model variables constant. The model-predicted systolic lumen diameter was reduced to $2.8 \pm 0.2 \mathrm{~mm}$ and the model-predicted distensibility increased only $21 \%$, not enough to explain the reported differences in $D$ between the Marfan and control groups measured in vivo. ${ }^{12,14}$ This suggests that that the intrinsic elastic properties of both Marfan patients and BAV patients differ from age-matched controls, and these differences are primarily responsible for reduced distensibility.

The relation between elastic properties and distensibility is also evident from our comparison of elastic properties at simulated diastolic and systolic stress states. Jeremy and coworkers ${ }^{14}$ found that distensibility decreased with age in both Marfan patient and control groups, consistent with the trends in model-predicted $D$ for our BAV and DA patient groups. Within the BAV group, the difference in circumferential stretch ratio at diastolic and systolic stress states decreased with age. In the Marfan patient group, the difference in circumferential stretch ratios between the diastolic and systolic states was small (Figure 5, F), despite the relatively large circumferential stretch ratio at the diastolic 
stress state. This can be attributed to greater nonlinearity in the biaxial elastic properties of aortic tissue from Marfan patient group than the BAV patient group (see Figure 2). Although the decrease in $D$ with age in our Marfan group was not significant, the younger Marfan patients we studied may have had more severe cardiovascular involvement, influencing the distensibility, or the small number of Marfan patients studied may have prevented us from observing age-related changes.

Our model results also suggest that altered distensibility alone does not affect the risk of rupture, since distensibility was not directly related to the level of circumferential stress. Nonetheless, it is possible that decreases in strength occur simultaneously with alterations in elastic properties, thereby indirectly influencing the risk of rupture.

Functional valve pathology did not appear to have a significant effect on either in vitro mechanical properties or in vivo stresses, although a type II error cannot be excluded. These findings further support the hypothesis that aortic dilatation in BAV patients is due to intrinsic material abnormalities rather than abnormal flow patterns. ${ }^{17,18}$

The severity of elastin fragmentation in our study group was similar to that previously reported by Schlatmann and Becker ${ }^{19}$ for dilated ascending aorta. Our observation that 4 of $6 \mathrm{BAV}$ patients with moderate or severe elastin fragmentation were in the younger age group is consistent with the suggestion by de $\mathrm{Sa}$ and colleagues ${ }^{3}$ that patients with more severe histopathologic changes may require earlier surgical intervention. The relative change in circumferential stretch ratio between the simulated diastolic and systolic states (Figure 5, $F)$ was significantly lower $(P=.014)$ in patients with moderate or severe elastin fragmentation compared with those with mild elastin fragmentation, suggesting that elastin fragmentation may be associated with altered elastic properties. There was no apparent relation between the severity of elastin fragmentation and functional valve pathology. The frequency of severe fibrosis and necrosis were both lower in our study than in the study by Schlatmann and Becker, ${ }^{19}$ and thus our data may not distinguish the effect of fibrosis on elastic properties.

\section{Limitations}

The principal limitation of this study is the lack of normal aorta for analysis. Although normal descending thoracic aorta would be available from thoracic organ transplant donors, excess ascending aorta is not generally available in the quantity required for these studies. Furthermore, the ascending and descending thoracic aortic segments have different wall structures ${ }^{20}$ and behave differently clinically, making this a poor control tissue. Limitations of the cylindrical model must also be acknowledged. In the cylindrical model it is assumed that the aorta is stretched by $20 \%$ axially throughout the cardiac cycle. If the actual values of axial stretch are higher, the axial stress would also increase. In the absence of experimental data, we have also assumed the same axial stretch for all patients regardless of age, basing our value on the studies of Learoyd and Taylor. ${ }^{11}$

Our model also assumes that wall thickness, elastic properties, and lumen diameter are uniform. Although this simplifies the model analysis, it neglects possible regional or local variations in wall thickness and elastic properties and the large axial variations in diameter in the dilated ascending aorta. We did not note obvious differences in wall thickness in our excised specimens, but local or regional variations in thickness may exist, altering local stresses. Our biaxial test method requires a relatively large specimen, making it difficult to test multiple specimens from different regions to determine whether regional variations in elastic properties are present. Finite element models of the ascending aorta that more accurately represent dilated aortic geometry, such as those of Grande and colleagues, ${ }^{21,22}$ may predict local stresses that exceed the mean values predicted by the cylindrical model. However, previous finite element models have been limited by a lack of measured material properties for the aneurysmal aortic wall. Thus, combining the in vitro material properties measured in this study and more detailed finite element model geometry should allow future models to more accurately predict wall stress distributions.

Although the magnitude of the opening angle has little effect on the wall-averaged circumferential stress presented in this study, it strongly affects the distribution of predicted circumferential stress through the wall. ${ }^{10}$ If the circumferential residual stress is neglected (ie, $\alpha=0^{\circ}$ ), the circumferential stress is highest near the inner wall. As the opening angle increases, the circumferential stress becomes more uniform through the wall. There is an opening angle at which the circumferential stress is nearly constant, but with larger opening angles the stress becomes higher toward the outer wall. A similar trend is seen in the distribution of predicted axial stress through the wall. This suggests that the large opening angles observed in our study may lead to higher stresses near the adventitia. However, transmural stress distributions are also influenced by nonhomogeneous material properties (ie, different properties for the media and adventitia). ${ }^{10,23}$ More detailed studies of the elastic properties of the individual layers will be needed to predict the transmural stress distribution; however, such studies are technically difficult.

\section{Conclusions}

In summary, our analysis indicates that mean circumferential stress in dilated ascending aorta increases linearly with blood pressure and diameter, supporting the clinical importance of blood pressure control and serial evaluation of aortic diameter. Decreasing wall strength with age may 
increase the risk of rupture or dissection consistent with clinical observations. Differences in elastic properties cause differences in model-predicted distensibilities associated with age and clinical group. Low model-predicted distensibility in Marfan patients is associated with severe elastic fragmentation. Functional valve pathology does not appear to influence elastic properties or distensibility, supporting the hypothesis that aortic dilatation in BAV patients is due to intrinsic abnormalities in the aortic wall rather than abnormal flow patterns.

We thank Sadaf Toor for performing data analysis and Nancy Nickerson and Marci Bailey for gathering patient information and obtaining patient consent. Aldi Kraja of the Division of Biostatistics at Washington University served as statistical consultant, and we appreciate his valuable input.

\section{References}

1. Davies RR, Goldstein LJ, Coady MA, et al. Yearly rupture or dissection rates for thoracic aortic aneurysms: simple prediction based on size. Ann Thorac Surg. 2002;73:17-27.

2. Halme T, Savunen T, Aho H, Vihersaari T, Penttinen R. Elastin and collagen in the aortic wall: changes in the Marfan syndrome and annuloaortic ectasia. Exp Mol Pathol. 1985;43:1-12.

3. de Sa M, Moshkovitz Y, Butany J, David TE. Histologic abnormalities of the ascending aorta and pulmonary trunk in patients with bicuspid aortic valve disease: clinical relevance to the Ross procedure. J Thorac Cardiovasc Surg. 1999;118:588-94.

4. Okamoto RJ, Wagenseil JE, DeLong WD, Peterson SJ, Kouchoukos NT, Sundt TM. Mechanical properties of dilated human ascending aorta. Ann Biomed Eng. 2002;30:624-35.

5. Schlatmann TJ, Becker AE. Histologic changes in the normal aging aorta: implications for dissecting aortic aneurysm. Am J Cardiol. 1977;39:13-20.

6. Liu SQ, Fung YC. Zero-stress states of arteries. J Biomech Eng. 1988;110:82-4.

7. von Maltzahn WW, Besdo D, Wiemer W. Elastic properties of arteries: a nonlinear two-layer cylindrical model. J Biomech. 1981;14:38997.

8. Chuong CJ, Fung YC. On residual stresses in arteries. J Biomech Eng. 1986;108:189-92.

9. Matsumoto T, Hayashi K. Stress and strain distribution in hypertensive and normotensive rat aorta considering residual strain. J Biomech Eng. 1996;118:62-73.

10. Peterson SJ, Okamoto RJ. Effect of residual stress and heterogeneity on circumferential stress in the arterial wall. J Biomech Eng. 2000; 122:454-6.

11. Learoyd BM, Taylor MG. Alterations with age in the viscoelastic properties of human arterial walls. Circ Res. 1966;18:278-92.

12. Hirata K, Triposkiadis F, Sparks E, Bowen J, Wooley CF, Boudoulas H. The Marfan syndrome: abnormal aortic elastic properties. J Am Coll Cardiol. 1991;18:57-63.

13. Juvonen T, Ergin MA, Galla JD, et al. Prospective study of the natural history of thoracic aortic aneurysms. Ann Thorac Surg. 1997;63:153345.

14. Jeremy RW, Huang H, Hwa J, McCarron H, Hughes CF, Richards JG. Relation between age, arterial distensibility, and aortic dilatation in the Marfan syndrome. Am J Cardiol. 1994;74:369-73.

15. Adams JN. Aortic distensibility and stiffness index measured by magnetic resonance imaging in patients with Marfan's syndrome. $\mathrm{Br}$ Heart J. 1995;73:265-9.

16. Nistri S, Sorbo MD, Basso C, Thiene G. Bicuspid aortic valve: abnormal aortic elastic properties. J Heart Valve Dis. 2002;11:369-73.

17. Pachulski RT, Weinberg AL, Chan KL. Aortic aneurysm in patients with functionally normal or minimally stenotic bicuspid aortic valve. Am J Cardiol. 1991;67:781-2.
18. Hahn RT, Roman MJ, Mogtader AH, Devereux RB. Association of aortic dilation with regurgitant, stenotic and functionally normal bicuspid aortic valves. J Am Coll Cardiol. 1992;19:283-8.

19. Schlatmann TJ, Becker AE. Pathogenesis of dissecting aneurysm of aorta. Comparative histopathologic study of significance of medial changes. Am J Cardiol. 1977;39:21-6.

20. Halloran BG, Davis VA, McManus BM, Lynch TG, Baxter BT. Localization of aortic disease is associated with intrinsic differences in aortic structure. J Surg Res. 1995;59:17-22.

21. Grande KJ, Cochran RP, Reinhall PG, Kunzelman KS. Mechanisms of aortic valve incompetence: finite element modeling of aortic root dilatation. Ann Thorac Surg. 2000;69:1851-7.

22. Grande KJ, Cochran RP, Reinhall PG, Kunzelman KS. Stress variations in the human aortic root and valve: the role of anatomic asymmetry. Ann Biomed Eng. 1998;26:534-45.

23. Taber LA, Humphrey JD. Stress-modulated growth, residual stress, and vascular heterogeneity. J Biomech Eng. 2001;123:528-35.

24. von Maltzahn WW, Warriyar RG, Keitzer WF. Experimental measurements of elastic properties of media and adventitia of bovine carotid arteries. J Biomech. 1984;17:839-47.

\section{Appendix}

\section{Material Law for Elastic Properties}

The elastic properties of the aortic tissue were represented by an incompressible, nonlinear, anisotropic pseudo strain energy function, $W^{24}$, with 4 material parameters $\left\{c, b_{1}, b_{2}, b_{3}\right\}$ :

$$
W=\frac{c}{2}(\exp Q-1) \text { where } Q=b_{1} E_{C}^{2}+b_{2} E_{Z}^{2}+2 b_{3} E_{C} E_{Z}
$$

In the absence of shear strains, circumferential $\left(\lambda ;_{c}\right)$ and axial $\left(\lambda_{z}\right)$ stretch ratios are related to the corresponding Lagrangian strain components $E_{c}=0.5 \cdot\left(\lambda_{c}{ }^{2}-1\right)$ and $E_{z}=0.5 \cdot\left(\lambda_{z}{ }^{2}-1\right)$. The Cauchy stress components are related to the partial derivatives of $W$ in Equation (A.1) with respect to the strain components and are given $b^{24}$ :

$$
\begin{aligned}
& \sigma_{c}-\sigma_{r}=\lambda_{c}^{2} c \exp (Q)\left(b_{1} E_{c}+b_{3} E_{Z}\right) \\
& \sigma_{Z}-\sigma_{r}=\lambda_{Z}^{2} c \exp (Q)\left(b_{3} E_{C}+b_{2} E_{Z}\right)
\end{aligned}
$$

For planar biaxial testing, the radial stress, $\sigma_{r}$, is 0 and the strains, circumferential stress, $\sigma_{c}$, and axial stress, $\sigma_{z}$, are constant through the thickness of the specimen.

\section{Cylindrical Model Equation}

In the cylindrical model, strain and stress components, including $\sigma_{r}$, are functions of radial position in the aortic wall, with the boundary condition $P_{o}=-\sigma_{r}=0$ at $r_{o}$. The internal pressure, $P_{i}$, is found by substituting Equation (A.2a) into the equation of radial equilibrium in cylindrical coordinates,

$$
\frac{d \sigma_{r}}{d r}+\frac{\sigma_{r}-\sigma_{c}}{r}=0
$$

and integrating numerically from $r_{i}$ to $r_{o}$ :

$$
P_{i}=\int_{r_{i}}^{r_{0}}\left(\lambda_{\mathrm{c}}^{2} c \exp (Q)\left(b_{1} E_{C}+b_{3} E_{Z}\right)\right) \frac{d r}{r}
$$

